\title{
Chirally rotated Schrödinger functional: non-perturbative tuning in the quenched approximation
}

\section{HU-EP-09/43 \\ SFB/CPP-09-85 \\ DESY 09-146 \\ LTH 841}

\author{
J. Gonzalez Lopez ${ }^{* a, b}$, K. Jansen ${ }^{b}$, D. B. Renner ${ }^{b}$ and A. Shindler ${ }^{c^{\dagger}}$ \\ ${ }^{a}$ Humboldt-Universität zu Berlin, Institut für Physik \\ Newtonstrasse 15, 12489 Berlin, Germany \\ ${ }^{b} \mathrm{NIC}, \mathrm{DESY}$ \\ Platanenallee 6, 15738 Zeuthen, Germany \\ ${ }^{c}$ Division of Theoretical Physics, University of Liverpool \\ Peach Street, Liverpool L69 7ZL, United Kingdom \\ E-mail: jenifer.gonzalez.lopez@desy.de
}

\begin{abstract}
The use of chirally rotated boundary conditions provides a formulation of the Schrödinger functional that is compatible with automatic $O(a)$ improvement of Wilson fermions in the bulk. The elimination of bulk $O(a)$ terms requires the non-perturbative tuning of the critical mass and one additional boundary counterterm. We present the results of such a tuning in the quenched approximation at three values of the renormalised gauge coupling and for a range of lattice spacings.
\end{abstract}

The XXVII International Symposium on Lattice Field Theory

July 26-31, 2009

Peking University, Beijing, China

\footnotetext{
* Speaker.

†Current address: Instituto de Física Teórica UAM/CSIC Universidad Autónoma de Madrid, Cantoblanco E-28049 Madrid, Spain
} 


\section{Introduction}

Obtaining precise physical results from lattice calculations requires a well controlled continuum limit and, for many quantities, non-perturbative renormalisation. Ideally the renormalisation scheme should be not only non-perturbative but also mass independent and preferably gauge invariant. Schrödinger functional (SF) schemes $[1,2,3]$ are known to fulfill these properties. Additionally, to ease the burden of taking the continuum limit, $O(a)$ improvement is highly desirable. However, to eliminate the many counterterms necessary when applying the standard $O(a)$ improvement program with Wilson fermions, we would like to capitalize on the automatic $O(a)$ improvement provided by maximally twisted mass fermions [4] (see [5] for a review). Unfortunately, bulk automatic $O(a)$ improvement with Wilson fermions and the standard SF (sSF) boundary conditions (BCs) are not compatible. $O(a)$ improvement is only possible introducing a number of additional bulk improvement counter-terms to the action and operators. Since there are extensive calculations with maximally twisted mass fermions $[6,7]$ it would be clearly desirable to employ the SF scheme while keeping automatic $O(a)$-improvement.

A new formulation of the SF has been developed in Ref. [8], which we will refer to as the chirally rotated SF $(\chi \mathrm{SF})$, that implements a SF scheme while maintaining automatic $O(a)$ improvement for massless Wilson fermions. The $\chi \mathrm{SF}$ is related (in the continuum) to the sSF by means of a non-singlet chiral transformation, i.e. they are equivalent in the continuum limit. However, when using massless Wilson fermions as a lattice regulator, $\chi \mathrm{SF} \mathrm{BCs}$ are invariant under a subgroup of the chiral symmetry transformations broken by the Wilson term (in contrast to sSF $\mathrm{BCs})$. As a result $\chi \mathrm{SF} \mathrm{BCs}$ are compatible with automatic $O(a)$ improvement.

The three-dimensional boundaries of the SF lead to an unavoidable dimension four boundary operator. Additionally, regulating the $\chi \mathrm{SF}$ with Wilson fermions induces the usual bulk mass operator as well as a dimension three boundary operator. The dimension four boundary operator is irrelevant, and hence the corresponding coefficient can be safely fixed by perturbation theory in order to eliminate the corresponding $O(a)$ boundary contributions. The bulk operator is relevant and is handled by the standard non-perturbative tuning of the bare quark mass, equivalently $\kappa$, to its critical value. The dimension three operator is also relevant and can spoil not only the automatic $O(a)$ improvement but also the universality of the continuum limit. This requires an additional non-perturbative tuning of one more counterterm, $z_{\mathrm{f}}$. However, having tuned both $\kappa$ and $z_{\mathrm{f}}$, all operators are automatically $O(a)$ improved and no further counterterms are necessary.

Here we present the non-perturbative tuning of $\kappa$ and $z_{\mathrm{f}}$ for the $\chi \mathrm{SF}$ in the quenched approximation. We demonstrate the feasibility of tuning both parameters simultaneously. In particular, the inclusion of the bulk dimension five operator, with corresponding counterterm $c_{\mathrm{sw}}$, as used in Ref. [9], is found to be unnecessary.

\section{Boundary conditions}

The $\chi \mathrm{SF}$ is related to the sSF by a non-singlet chiral transformation, $\chi=\exp \left(-i \pi \gamma_{5} \tau^{3} / 4\right) \psi$, where $\psi$ is the fermion doublet in the $N_{\mathrm{f}}=2$ standard formulation, $\chi$ is the corresponding doublet in the rotated basis and $\tau^{3}$ is a Pauli matrix. This field transformation maps the sSF BCs to the $\chi \mathrm{SF}$ 
BCs,

$$
\begin{array}{ll}
\left.Q_{+} \chi(x)\right|_{x_{0}=0}=0 & \left.Q_{-} \chi(x)\right|_{x_{0}=T}=0 \\
\left.\bar{\chi}(x) Q_{+}\right|_{x_{0}=0}=0 & \left.\bar{\chi}(x) Q_{-}\right|_{x_{0}=T}=0,
\end{array}
$$

where $T$ is the Euclidean time extent and $Q_{ \pm}$are projectors given by

$$
Q_{ \pm}=\frac{1}{2}\left(\mathbb{1} \pm i \gamma_{0} \gamma_{5} \tau^{3}\right)
$$

Thus the $Q_{ \pm}$are simply the chirally rotated projectors corresponding to the sSF projectors, $P_{ \pm}=$ $1 / 2\left(1 \pm \gamma_{0}\right)$. However, once the theory is regularised on the lattice, we must ensure that the BCs in (2.1) are in fact recovered in the continuum limit. Using orbifolding techniques, it was shown that the BCs can be implemented at finite lattice spacing by a simple modification of the standard Wilson-Dirac operator, $D_{\mathrm{W}}$, near the time boundaries [10]. The resulting action is

$$
S=a^{4} \sum_{x_{0}=0}^{T} \sum_{\tilde{\mathrm{x}}} \bar{\chi}(x)\left(\mathscr{D}_{\mathrm{W}}+m_{0}\right) \chi(x)
$$

and the modified Wilson-Dirac operator is given by

$$
a \mathscr{D}_{\mathrm{W}} \chi(x)= \begin{cases}-U(x, 0) P_{-} \chi(x+a \hat{0})+\left(a K+i \gamma_{5} \tau^{3} P_{-}\right) \chi(x) & \text { if } \quad x_{0}=0 \\ a D_{\mathrm{W}} \chi(x) & \text { if } 0<x_{0}<T \\ \left(a K+i \gamma_{5} \tau^{3} P_{+}\right) \chi(x)-U^{\dagger}(x-a \hat{0}, 0) P_{+} \chi(x-a \hat{0}) & \text { if } \quad x_{0}=T\end{cases}
$$

where $K$ is the time-diagonal contribution to $D_{\mathrm{W}}$.

\section{Boundary counterterms}

To ensure the correct continuum limit, we must account for all relevant operators allowed by the symmetries of the action above. This means dimension four or less for the bulk action. There is one such operator, $\bar{\chi} \chi$, and the corresponding counterterm is the term proportional to the critical quark mass, $m_{\mathrm{cr}}$, or equivalently $\kappa_{\mathrm{cr}}$. This is the standard operator that is present for all Wilson actions due to the breaking of chiral symmetry by the Wilson term.

Similarly, we must include all permitted boundary operators of dimension three or less. Again, the one allowed operator is $\bar{\chi} \chi$ [8], which gives rise to the following counterterm to the lattice action,

$$
\delta S_{3}=\left(z_{\mathrm{f}}-1\right) a^{3} \sum_{\vec{x}}\left(\left.\bar{\chi} \chi\right|_{x_{0}=0}+\left.\bar{\chi} \chi\right|_{x_{0}=T}\right) .
$$

Such an operator would be forbidden in the continuum action, but the reduced symmetries of the Wilson action do not allow us to exclude this operator on the lattice. The presence of $\delta S_{3}$ can then be understood as necessary to restore the symmetries broken by the Wilson term in the continuum limit. The fact that it is a relevant operator implies that we must compute the bare coupling dependence of $z_{\mathrm{f}}$ non-perturbatively, just as for $\kappa$. 
Furthermore, we must examine those irrelevant operators that lead to $O(a)$ contributions. In the bulk, there is the dimension five Sheikholeslami-Wohlert term, but automatic $O(a)$ improvement eliminates the need for this operator. Yet, there does remain an $O(a)$ contribution from the boundary due to the irrelevant dimension four operator [9],

$$
\delta S_{4}=\left(d_{\mathrm{s}}-1\right) a^{4} \sum_{\vec{x}}\left(\left.\bar{\chi} \gamma_{k} D_{k} \chi\right|_{x_{0}=0}+\left.\bar{\chi} \gamma_{k} D_{k} \chi\right|_{x_{0}=T}\right) .
$$

Such a contribution is present in all SF formulations [3] and is not due to the particular lattice action or BCs we have chosen. In fact, $d_{\mathrm{s}}$ plays a role that is analogous to the $\tilde{c}_{t}$ counterterm in the sSF [11]. Given that $\delta S_{4}$ is an irrelevant operator, $d_{s}$ can be computed in perturbation theory. For the investigation presented here, we simply use the tree-level value of $1 / 2$.

\section{Tuning conditions}

The non-perturbative determination of $\kappa$ and $z_{\mathrm{f}}$ requires imposing conditions at finite lattice spacing that ensure the restoration of all expected symmetries in the continuum limit: parity and flavour symmetries in the $\chi$ - basis ${ }^{1}$. Moreover, these conditions should be imposed at each lattice spacing while fixing a suitable renormalised quantity. In this work, we keep the renormalised SF coupling, $\bar{g}$, fixed. This is equivalent to fixing the physical size of the box, $L$. All other dimensionful quantities must scale with $L$, so we choose $T=L$, evaluate all correlation functions at $x_{0}=T / 2$ and use periodic boundary conditions with $\theta=0$.

Before specifying the tuning conditions, we define the following boundary to bulk correlation functions

$$
g_{\mathrm{A}_{ \pm}}^{a b}\left(x_{0}\right)=-\left\langle A_{0}^{a}(x) \mathscr{Q}_{ \pm}^{b}\right\rangle \quad g_{\mathrm{P}_{ \pm}}^{a b}\left(x_{0}\right)=-\left\langle P^{a}(x) \mathscr{Q}_{ \pm}^{b}\right\rangle
$$

where the boundary operator, $\mathscr{Q}_{ \pm}^{a}$, is defined for the $x_{0}=0$ boundary by

$$
\mathscr{Q}_{ \pm}^{a}=a^{6} \sum_{\vec{y}, \vec{z}} \bar{\zeta}(\vec{y}) \gamma_{5} \frac{1}{2} \tau^{a} Q_{ \pm} \zeta(\vec{z}) e^{i \vec{p}(\vec{y}-\vec{z})}
$$

the bulk operators $A_{\mu}^{a}(x)$ and $P^{a}(x)$ are the axial current and pseudoscalar density in the $\chi$-basis, and the boundary fields for $x_{0}=0$ are defined as

$$
\zeta(\vec{x})=\left.U\left(x_{0}-a, \vec{x} ; 0\right) \chi(x)\right|_{x_{0}=a} \quad \bar{\zeta}(\vec{x})=\left.\bar{\chi}(x) U^{\dagger}\left(x_{0}-a, \vec{x} ; 0\right)\right|_{x_{0}=a} .
$$

To tune $\kappa$ to its critical value, we adopt the standard procedure of imposing a vanishing PCAC mass. To tune $z_{\mathrm{f}}$, we require the $\gamma_{5} \tau_{1}$-odd correlation function $g_{\mathrm{A}_{-}}^{11}$ to vanish,

$$
m_{\mathrm{PCAC}} \equiv \frac{\partial_{0}^{1 \text { latt }} g_{\mathrm{A}_{-}}^{11}(T / 2)}{2 g_{\mathrm{P}_{-}}^{11}(T / 2)}=0 \quad g_{\mathrm{A}_{-}} \equiv g_{\mathrm{A}_{-}}^{11}(T / 2)=0 .
$$

The second condition in particular is sensitive to the symmetries broken by the lattice action (2.2), and both conditions together ensure that in the continuum limit all broken symmetries are indeed restored. Imposing different symmetry restoration conditions would give rise to different values of $\kappa$ and $z_{\mathrm{f}}$ that would differ amongst themselves by cutoff effects. It will be important to study the sensitivity of $\kappa$ and $z_{\mathrm{f}}$ to the particular definitions used in order to better understand the intrinsic uncertainty in the determination of these counterterms.

\footnotetext{
${ }^{1}$ We recall that in the $\chi$ - basis parity and flavour symmetries take a slightly different form (see ref. [5] for a discussion about the dependence of the symmetries on the basis adopted).
} 


\begin{tabular}{|r|c|l|l|l|}
\hline$L / a$ & $\beta$ & $z_{\mathrm{f}}^{*}(\chi \mathrm{SF})$ & \multicolumn{1}{|c|}{$\kappa_{\mathrm{cr}}(\chi \mathrm{SF})$} & \multicolumn{1}{|c|}{$\kappa_{\mathrm{cr}}(\mathrm{sSF})$} \\
\hline \multicolumn{5}{|c|}{ Tuning at a hadronic scale, $\mu \sim 300 \mathrm{MeV}$} \\
\hline 8 & 6.0219 & $1.8090(32)$ & $0.153530(24)$ & $0.153371(10)$ \\
10 & 6.1628 & $1.7920(30)$ & $0.152134(17)$ & $0.152012(7)$ \\
12 & 6.2885 & $1.7664(51)$ & $0.150815(22)$ & $0.150752(10)$ \\
16 & 6.4956 & $1.7212(83)$ & $0.148945(25)$ & $0.148876(13)$ \\
\hline \multicolumn{5}{|c|}{ Tuning at an intermediate scale, $\mu \sim 1 \mathrm{GeV}$} \\
\hline 8 & 7.0197 & $1.5467(15)$ & $0.144501(13)$ & $0.144454(7)$ \\
12 & 7.3551 & $1.5126(23)$ & $0.143113(12)$ & $0.143113(6)$ \\
16 & 7.6101 & $1.4942(37)$ & $0.142112(13)$ & $0.142107(6)$ \\
\hline \multicolumn{5}{|c|}{ Tuning at a perturbative scale, $\mu \sim 30 \mathrm{GeV}$} \\
\hline 8 & 10.3000 & $1.29730(67)$ & $0.1354609(54)$ & $0.135457(5)$ \\
12 & 10.6086 & $1.2954(11)$ & $0.1351758(56)$ & $0.135160(4)$ \\
16 & 10.8910 & $1.2858(15)$ & $0.1348440(61)$ & $0.134849(6)$ \\
\hline
\end{tabular}

Table 1: Tuning results at a hadronic, intermediate and perturbative scale. We give the critical values, $z_{\mathrm{f}}^{*}$ and $\kappa_{\mathrm{cr}}$, calculated in this work for the $\chi \mathrm{SF}$. For reference, we also give $\kappa_{\mathrm{cr}}$ for the sSF $[12,13,14]$.

\section{Tuning results}

To check the practicality of tuning both $\kappa$ and $z_{\mathrm{f}}$ non-perturbatively for the $\chi \mathrm{SF}$, we perform the tuning at three values of the renormalisation scale $\mu=1 / L$, corresponding to a hadronic $\left(\bar{g}^{2}\right.$ fixed with $\left.L=1.436 r_{0}\right)$, an intermediate $\left(\bar{g}^{2}=2.4484\right)$ and a perturbative $\left(\bar{g}^{2}=0.9944\right)$ scale. The results at these three points are summarised in Tab. 1. We now briefly explain the procedure we used to perform the tuning, showing examples from our most difficult point at the hadronic scale and for the smallest lattice, $L / a=8$.

The values of $\beta$ used are given in Tab. 1 and are taken from Ref. [15]. The tuning is performed in several steps. First, we calculate $m_{\mathrm{PCAC}}$ and $g_{\mathrm{A}-}$ at four values of $z_{\mathrm{f}}$, and for each value of $z_{\mathrm{f}}$, we use four values of $\kappa$, thus giving 16 pairs of $\kappa$ and $z_{\mathrm{f}}$. This allows us to determine $g_{A_{-}}$as a function of $m_{\mathrm{PCAC}}$ for each value of $z_{\mathrm{f}}$, as illustrated in Fig. 1 . For each value of $z_{\mathrm{f}}$, we perform a linear interpolation of $g_{\mathrm{A}-}$ in terms of $m_{\mathrm{PCAC}}$ to the point $m_{\mathrm{PCAC}}=0$. This determines the values of $g_{\mathrm{A}-}$ at $m_{\mathrm{PCAC}}=0$ for each of the four values of $z_{\mathrm{f}}$, as shown in Fig. 2. We now interpolate these values of $g_{\mathrm{A}-}$ as a function of $z_{\mathrm{f}}$ to the point of vanishing $g_{A_{-}}$, thus giving us the critical value $z_{\mathrm{f}}^{*}$.

Next we determine $\kappa_{\mathrm{cr}}$. Using the same 16 pairs of $\kappa$ and $z_{\mathrm{f}}$, we calculate $m_{\mathrm{PCAC}}$ as a function of $\kappa$ for each $z_{\mathrm{f}}$. This is shown in Fig. 3. Note that $m_{\text {PCAC }}$ has a very mild dependence on $z_{\mathrm{f}}$, so the four curves at fixed $z_{\mathrm{f}}$ are nearly indistinguishable. Interpolating in $\kappa$ to the point of vanishing PCAC mass, we obtain the critical values of $\kappa$ at each $z_{\mathrm{f}}$. The resulting values of $\kappa$ as a function of $z_{\mathrm{f}}$ are shown in Fig. 4. We now interpolate these results in $z_{\mathrm{f}}$ to the previously determined value of $z_{\mathrm{f}}^{*}$, thus determining the value of $\kappa_{\mathrm{cr}}$.

A key observation of this work is the mild dependence of $m_{\mathrm{PCAC}}$ on $z_{\mathrm{f}}$, at least in the region near $\kappa_{\mathrm{cr}}$ and $z_{\mathrm{f}}^{*}$. You can easily see this in Fig. 3. The consequence of this is clear in Fig. 4: the determination of $\kappa_{\mathrm{cr}}$ also has a weak dependence on $z_{\mathrm{f}}^{*}$ and the errors of both are relatively 


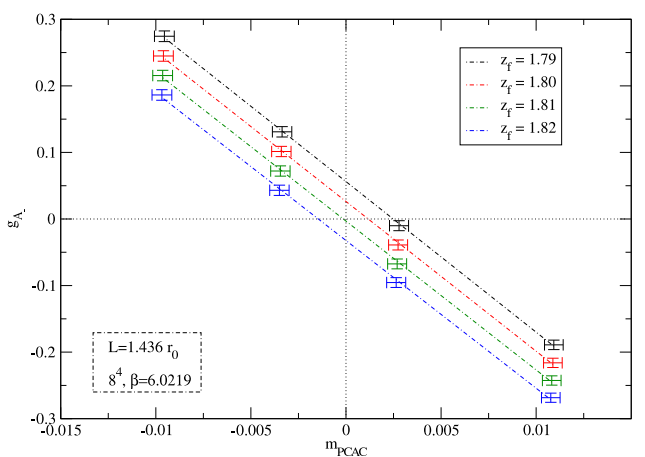

Figure 1: Plot of $g_{\mathrm{A}-}$ versus $m_{\mathrm{PCAC}}$.

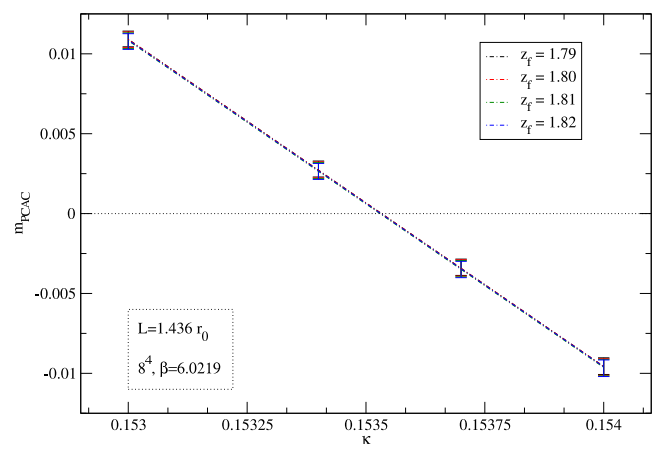

Figure 3: Plot of $m_{\mathrm{PCAC}}$ versus $\kappa$.

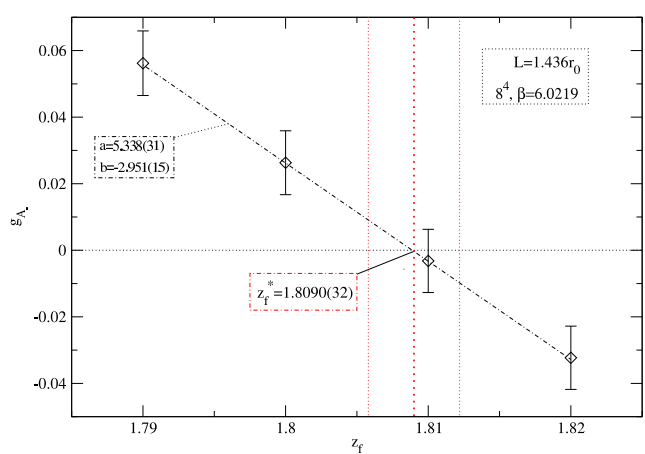

Figure 2: Plot of $g_{\mathrm{A}-}$ versus $z_{\mathrm{f}}$.

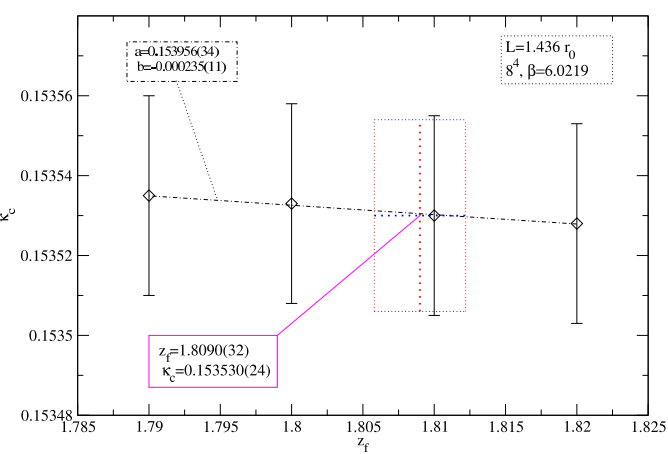

Figure 4: Plot of $\kappa_{\mathrm{cr}}$ versus $z_{\mathrm{f}}$.

independent. If this behaviour persists with dynamical calculations, it could ease the numerical effort necessary to perform the tuning, thus reducing the number of required simulations.

\section{Conclusions}

We have presented the results of the non-perturbative tuning of $\kappa$ and $z_{\mathrm{f}}$ for the $\chi \mathrm{SF}$ at three physical scales and for a range of lattice spacings. This demonstrates that the tuning of these two coefficients is indeed feasible, at least in the quenched approximation. Moreover, we observe that the tuning of $z_{\mathrm{f}}$ and $\kappa$ are nearly independent. Note that even with non-improved Wilson fermions in the bulk, $\kappa$ and $z_{\mathrm{f}}$ are the only parameters that must be tuned within the $\chi \mathrm{SF}$ setup in order to guarantee bulk automatic $O(a)$ improvement, thus eliminating the need for the bulk counterterm, $c_{\mathrm{sw}}$, and for the many operator improvement coefficients necessary in the sSF.

Our next step is to perform an universality test of this formulation as well as a demonstration that automatic $O(a)$ improvement holds. This can be done by reproducing a variety of quantities already computed in the standard setup. A natural candidate would be the computation of the stepscaling function of the pseudoscalar renormalisation factor, $Z_{\mathrm{P}}$, which could be compared to the 
results of [15]. We recall that the $\chi \mathrm{SF}$ and the sSF are equivalent in the continuum limit, therefore, it is not necessary to recompute the entire evolution of an operator. The only quantity that must be recomputed is the renormalisation factor at the most non-perturbative scale.

We also plan to explore whether the value of $\kappa_{\text {cr }}$ determined from the finite volume simulations can be used in large volume, preserving the nice scaling behaviour obtained in Refs. [16, 17], without the need for a large volume determination of $\kappa_{\mathrm{cr}}$. A lattice perturbation theory computation of $d_{\mathrm{s}}$ and $z_{\mathrm{f}}$ is also planned. The final goal is to perform dynamical simulations.

\section{Acknowledgments}

We thank S. Sint and B. Leder for many discussions and the private communication of the unpublished results of ref. [10]. We also acknowledge the support of the computer center in DESYZeuthen and the NW-grid in Lancaster.

\section{References}

[1] M. Lüscher et al., Nucl. Phys. B384 (1992) 168 [hep-lat/9207009].

[2] S. Sint, Nucl. Phys. B421 (1994) 135 [hep-lat/9312079].

[3] M. Lüscher, JHEP 05 (2006) 042 [hep-lat / 0603029 ].

[4] R. Frezzotti and G.C. Rossi, JHEP 08 (2004) 007 [hep-lat / 0306014 ].

[5] A. Shindler, Phys.Rept. 461 (2008) 37 [arXiv: 0707 . 4093].

[6] ETM Collaboration, P. Boucaud et al., Phys. Lett. B650, 304 (2007).

[7] ETM Collaboration, P. Boucaud et al., Comput. Phys. Commun. 179, 695 (2008).

[8] S. Sint, PoS (LAT2005) 235 [hep-lat/0511034].

[9] S. Sint, Talk at Lattice 2008; B. Leder, Talk at Lattice 2008

[10] S. Sint, In preparation.

[11] M. Lüscher et al., Nucl. Phys. B478 (1996) 365 [hep-lat/9605038].

[12] M. Guagnelli et al., JHEP 03 (2006) 088 [hep-lat / 0505002 ].

[13] M. Guagnelli et al., Nucl. Phys. B664 (2003) [hep-lat/0303012].

[14] M. Guagnelli et al., JHEP 05 (2004) 001 [hep-lat/ 0402 022].

[15] S. Capitani, M. Luscher, R. Sommer, H. Wittig, Nucl. Phys. B544 (1999) [hep-lat/9810063].

[16] K. Jansen et al., JHEP 09 (2005) 071 [hep-lat / 0507010 ].

[17] ETM Collaboration, P. Dimopoulos et al., arXiv:0810.2873 [hep-lat]. 